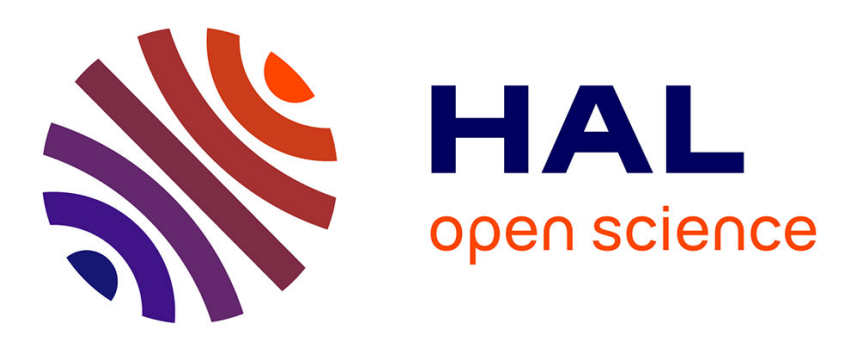

\title{
Energy and regularity dependent stability estimates for the Gel'fand inverse problem in multidimensions
}

\author{
Mikhail Isaev, Roman Novikov
}

\section{To cite this version:}

Mikhail Isaev, Roman Novikov. Energy and regularity dependent stability estimates for the Gel'fand inverse problem in multidimensions. Journal of Inverse and Ill-posed Problems, 2012, 20 (3), pp.313325. hal-00689636

\section{HAL Id: hal-00689636 https://hal.science/hal-00689636}

Submitted on 19 Apr 2012

HAL is a multi-disciplinary open access archive for the deposit and dissemination of scientific research documents, whether they are published or not. The documents may come from teaching and research institutions in France or abroad, or from public or private research centers.
L'archive ouverte pluridisciplinaire HAL, est destinée au dépôt et à la diffusion de documents scientifiques de niveau recherche, publiés ou non, émanant des établissements d'enseignement et de recherche français ou étrangers, des laboratoires publics ou privés. 


\title{
Energy and regularity dependent stability estimates for the Gel'fand inverse problem in multidimensions
}

\author{
M.I. Isaev and R.G. Novikov
}

\begin{abstract}
We prove new global Hölder-logarithmic stability estimates for the Gel'fand inverse problem at fixed energy in dimension $d \geq 3$. Our estimates are given in uniform norm for coefficient difference and related stability efficiently increases with increasing energy and/or coefficient regularity. Comparisons with preceeding results in this direction are given.
\end{abstract}

\section{Introduction}

We consider the Schrödinger equation

$$
-\Delta \psi+v(x) \psi=E \psi, \quad x \in D,
$$

where

$$
\begin{aligned}
& D \text { is an open bounded domain in } \mathbb{R}^{d}, d \geq 2, \\
& \text { with } \partial D \in C^{2}, \\
& \qquad v \in \mathbb{L}^{\infty}(D) .
\end{aligned}
$$

Consider the map $\hat{\Phi}=\hat{\Phi}(E)$ such that

$$
\hat{\Phi}(E)\left(\left.\psi\right|_{\partial D}\right)=\left.\frac{\partial \psi}{\partial \nu}\right|_{\partial D}
$$

for all sufficiently regular solutions $\psi$ of (1.1) in $\bar{D}=D \cup \partial D$, where $\nu$ is the outward normal to $\partial D$. Here we assume also that

$$
E \text { is not a Dirichlet eigenvalue for operator }-\Delta+v \text { in } D \text {. }
$$

The map $\hat{\Phi}=\hat{\Phi}(E)$ is called the Dirichlet-to-Neumann map and is considered as boundary measurements.

We consider the following inverse boundary value problem for equation (1.1):

Problem 1.1. Given $\hat{\Phi}$ for some fixed $E$, find $v$.

This problem can be considered as the Gelfand inverse boundary value problem for the Schrödinger equation at fixed energy (see [10], [23]). At zero energy this problem can be considered also as a generalization of the Calderon problem of the electrical impedance tomography (see [6], [23]). Problem 1.1 can be also 
considered as an example of ill-posed problem: see [18], [4] for an introduction to this theory.

Problem 1.1 includes, in particular, the following questions: (a) uniqueness, (b) reconstruction, (c) stability.

Global uniqueness results and global reconstruction methods for Problem 1.1 were given for the first time in [23] in dimension $d \geq 3$ and in [5] in dimension $d=2$.

Global logarithmic stability estimates for Problem 1.1 were given for the first time in [1] in dimension $d \geq 3$ and in [30] in dimension $d=2$. A principal improvement of the result of [1] was given recently in [29] (for the zero energy case): stability of [29] optimally increases with increasing regularity of $v$.

For the Calderon problem (of the electrical impedance tomography) in its initial formulation the global uniqueness was firstly proved in [36] for $d \geq 3$ and in [21] for $d=2$. Global logarithmic stability estimates for this problem were given for the first time in [1] for $d \geq 3$ and [19] for $d=2$. Principal increasing of global stability of [1], [19] for the regular coefficient case was found in [29] for $d \geq 3$ and [34] for $d=2$.

In addition, for the case of piecewise constant or piecewise real analytic conductivity the first uniqueness results for the Calderon problem in dimension $d \geq 2$ were given in [7], [16]. Lipschitz stability estimate for the case of piecewise constant conductivity was proved in [2] and additional studies in this direction were fulfilled in [33].

Due to [20] the logarithmic stability results of [1], [19] with their principal effectivization of [29], [34] are optimal (up to the value of the exponent). An extention of the instability estimates of [20] to the case of the non-zero energy as well as to the case of Dirichlet-to-Neumann map given on the energy intervals was given in [12].

On the other hand, it was found in [25], [26] (see also [28], [31]) that for inverse problems for the Schrödinger equation at fixed energy $E$ in dimension $d \geq 2$ (like Problem 1.1) there is a Hölder stability modulo an error term rapidly decaying as $E \rightarrow+\infty$ (at least for the regular coefficient case). In addition, for Problem 1.1 for $d=3$, global energy dependent stability estimates changing from logarithmic type to Hölder type for high energies were given in [15]. However, there is no efficient stability increasing with respect to increasing coefficient regularity in these results of [15]. An additional study, motivated by [15], [29], was given in [22].

In the present work we give new global Hölder-logarithmic stability estimates for Problem 1.1 in dimension $d \geq 3$ for the regular coefficient case, see Theorem 2.1 and Remark 2.6. Our estimates are given in uniform norm for coefficient difference and related stability efficiently increases with increasing energy and/or coefficient regularity. In particular cases, our new estimates become coherent (although less strong) with respect to results of [29], [26], see Remarks 2.2, 2.3. In general, our new estimates give some synthesis of several important preceeding results.

\section{Stability estimates}

In this section we assume for simplicity that

$$
v \in W^{m, 1}\left(\mathbb{R}^{d}\right) \text { for some } m>d, \operatorname{supp} v \subset D,
$$


where

$$
W^{m, 1}\left(\mathbb{R}^{d}\right)=\left\{v: \partial^{J} v \in L^{1}\left(\mathbb{R}^{d}\right),|J| \leq m\right\}, m \in \mathbb{N} \cup 0,
$$

where

$$
J \in(\mathbb{N} \cup 0)^{d},|J|=\sum_{i=1}^{d} J_{i}, \partial^{J} v(x)=\frac{\partial^{|J|} v(x)}{\partial x_{1}^{J_{1}} \ldots \partial x_{d}^{J_{d}}}
$$

Let

$$
\|v\|_{m, 1}=\max _{|J| \leq m}\left\|\partial^{J} v\right\|_{L^{1}\left(\mathbb{R}^{d}\right)}
$$

Let

$$
\text { \|A\| denote the norm of an operator }
$$

$$
A: \mathbb{L}^{\infty}(\partial D) \rightarrow \mathbb{L}^{\infty}(\partial D) .
$$

We recall that if $v_{1}, v_{2}$ are potentials satisfying (1.3), (1.5) for some fixed $E$, then

$$
\hat{\Phi}_{2}(E)-\hat{\Phi}_{1}(E) \text { is a compact operator in } \mathbb{L}^{\infty}(\partial D),
$$

where $\hat{\Phi}_{1}, \hat{\Phi}_{2}$ are the DtN maps for $v_{1}, v_{2}$, respectively, see [23], [27]. Note also that $(2.1) \Rightarrow(1.3)$.

Let

$$
s_{0}=\frac{m-d}{m}, \quad s_{1}=\frac{m-d}{d}, \quad s_{2}=m-d .
$$

Theorem 2.1. Let $D$ satisfy (1.2), where $d \geq 3$. Let $v_{1}, v_{2}$ satisfy (2.1) and (1.5) for some fixed real $E$. Let $\left\|v_{j}\right\|_{m, 1} \leq N, j=1,2$, for some $N>0$. Let $\hat{\Phi}_{1}(E)$ and $\hat{\Phi}_{2}(E)$ denote the DtN maps for $v_{1}$ and $v_{2}$, respectively. Then

$$
\left\|v_{2}-v_{1}\right\|_{L^{\infty}(D)} \leq C_{1}\left(\ln \left(3+\delta^{-1}\right)\right)^{-s}, \quad 0<s \leq s_{1},
$$

where $C_{1}=C_{1}(N, D, m, s, E)>0, \delta=\left\|\hat{\Phi}_{2}(E)-\hat{\Phi}_{1}(E)\right\|$ is defined according to (2.5). In addition, for $E \geq 0, \tau \in(0,1)$ and any $s \in\left[0, s_{1}\right]$,

$$
\left\|v_{2}-v_{1}\right\|_{L^{\infty}(D)} \leq C_{2}(1+\sqrt{E}) \delta^{\tau}+C_{3}(1+\sqrt{E})^{s-s_{1}}\left(\ln \left(3+\delta^{-1}\right)\right)^{-s},
$$

where $C_{2}=C_{2}(N, D, m, \tau)>0$ and $C_{3}=C_{3}(N, D, m, \tau)>0$.

Remark 2.1. Estimate (2.8) for $s=s_{0}$ is a variation of the result of [1] (see also [29], [13]). One can see that estimate (2.8), $s=s_{1}$, of Theorem 2.1 is more strong (as much as $s_{1}$ is greater than $s_{0}$ ) than the aforementioned result going back to [1].

Remark 2.2. Estimate (2.8) for $s=s_{2}, E=0, d=3$ was proved in [29]. One can see that this estimate of [29] is more strong (as much as $s_{2}$ is greater than $s_{1}$ ) than estimate $(2.8), s=s_{1}$, of Theorem 2.1 for $E=0, d=3$.

Remark 2.3. Using results of [26] one can obtain estimate (2.9) for $s=0$, $d=3$, with $s_{2}$ in place of $s_{1}$, for sufficiently great $E$ with respect to $N$. One can see that for this particular case the aforementioned corollary of [26] is more strong (as much as $s_{2}$ is greater than $s_{1}$ ) than estimate (2.9) of Theorem 2.1.

Remark 2.4. In a similar way with results of [13], [14], estimates (2.8), (2.9) can be extended to the case when we do not assume that condition (1.5) is fulfiled and consider an appropriate impedance boundary map instead of the Dirichlet-to-Neumann map. 
Remark 2.5. Concerning two-dimensional analogs of results of Theorem 2.1, see [25], [31], [34], [35].

Remark 2.6. Actually, in the proof of Theorem 2.1 we obtain the following estimate (see formula (4.19)):

$$
\left\|v_{1}-v_{2}\right\|_{\mathbb{L}^{\infty}(D)} \leq C_{4} \sqrt{E+\rho^{2}} e^{2 \rho L} \delta+C_{5}\left(E+\rho^{2}\right)^{-s_{1} / 2},
$$

where $L=\max _{x \in \partial D}|x|, C_{4}=C_{4}(N, D, m)>0, C_{5}=C_{5}(N, D, m)>0$ and parameter $\rho>0$ is such that $E+\rho^{2}$ is sufficiently large: $E+\rho^{2} \geq C_{6}(N, D, m)$. Estimates of Theorem 2.1 follow from estimate (2.10).

The proof of Theorem 2.1 and estimate (2.10) is given in Section 4 and is based on results recalled in Section 3. Actually, this proof is technically very similar to the proof of estimate (2.8) for $s=s_{0}$, see [1], [29], [13]. Possibility of such a proof of estimate (2.8) for $s=s_{1}, E=0$ was mentioned, in particular, in [32].

\section{$3 \quad$ Faddeev functions}

We consider the Faddeev functions $G, \psi, h$ (see [8], [9], [11], [23]):

$$
\begin{gathered}
G(x, k)=e^{i k x} g(x, k), \quad g(x, k)=-(2 \pi)^{-d} \int_{\mathbb{R}^{d}} \frac{e^{i \xi x} d \xi}{\xi^{2}+2 k \xi}, \\
\psi(x, k)=e^{i k x}+\int_{\mathbb{R}^{d}} G(x-y, k) v(y) \psi(y, k) d y,
\end{gathered}
$$

where $x \in \mathbb{R}^{d}, k \in \mathbb{C}^{d}, \operatorname{Im} k \neq 0, d \geq 3$,

$$
h(k, l)=(2 \pi)^{-d} \int_{\mathbb{R}^{d}} e^{-i l x} v(x) \psi(x, k) d x,
$$

where

$$
k, l \in \mathbb{C}^{d}, k^{2}=l^{2}, \operatorname{Im} k=\operatorname{Im} l \neq 0 .
$$

One can consider (3.2), (3.3) assuming that

$$
\begin{array}{r}
v \text { is a sufficiently regular function on } \mathbb{R}^{d} \\
\text { with suffucient decay at infinity. }
\end{array}
$$

For example, in connection with Problem 1.1, one can consider (3.2), (3.3) assuming that

$$
v \in \mathbb{L}^{\infty}(D), \quad v \equiv 0 \text { on } \mathbb{R} \backslash D .
$$

We recall that (see [8], [9], [11], [23]):

- The function $G$ satisfies the equation

$$
\left(\Delta+k^{2}\right) G(x, k)=\delta(x), \quad x \in \mathbb{R}^{d}, \quad k \in \mathbb{C}^{d} \backslash \mathbb{R}^{d} ;
$$


- Formula (3.2) at fixed $k$ is considered as an equation for

$$
\psi=e^{i k x} \mu(x, k),
$$

where $\mu$ is sought in $\mathbb{L}^{\infty}\left(\mathbb{R}^{d}\right)$;

- As a corollary of (3.2), (3.1), (3.7), $\psi$ satisfies (1.1) for $E=k^{2}$;

- The Faddeev functions $G, \psi, h$ are (non-analytic) continuation to the complex domain of functions of the classical scattering theory for the Schrödinger equation (in particular, $h$ is a generalized "scattering"' amplitude).

In addition, $G, \psi, h$ in their zero energy restriction, that is for $E=0$, were considered for the first time in [3]. The Faddeev functions $G, \psi, h$ were, actually, rediscovered in [3].

Let

$$
\begin{array}{r}
\Sigma_{E}=\left\{k \in \mathbb{C}^{d}: k^{2}=k_{1}^{2}+\ldots+k_{d}^{2}=E\right\}, \\
\Theta_{E}=\left\{k \in \Sigma_{E}, l \in \Sigma_{E}: \operatorname{Im} k=\operatorname{Im} l\right\}, \\
|k|=\left(|\operatorname{Re} k|^{2}+|\operatorname{Im} k|^{2}\right)^{1 / 2} .
\end{array}
$$

Under the assumptions of Theorem 2.1, we have that:

$$
\mu(x, k) \rightarrow 1 \quad \text { as } \quad|k| \rightarrow \infty
$$

and, for any $\sigma>1$,

$$
|\mu(x, k)| \leq \sigma \quad \text { for } \quad|k| \geq r_{1}(N, D, m, \sigma),
$$

where $x \in \mathbb{R}^{d}, k \in \Sigma_{E}$;

$$
\begin{array}{r}
\hat{v}(p)=\begin{array}{l}
\lim _{\substack{k, l) \in \in \Theta_{E}, k-l=p \\
|\operatorname{mm} k|=|\operatorname{Im} l| \rightarrow \infty}} h(k, l) \quad \text { for any } p \in \mathbb{R}^{d}, \\
|\hat{v}(p)-h(k, l)| \leq \frac{c_{1}(D, m) N^{2}}{\left(E+\rho^{2}\right)^{1 / 2}} \\
|\operatorname{Im} k|=|\operatorname{Im} l|=\rho, \quad E+\rho^{2} \geq r_{2}(N, D, m), \\
p^{2} \leq 4\left(E+\rho^{2}\right),
\end{array}
\end{array}
$$

where

$$
\hat{v}(p)=(2 \pi)^{-d} \int_{\mathbb{R}^{d}} e^{i p x} v(x) d x, \quad p \in \mathbb{R}^{d} .
$$

Results of the type (3.10), (3.11) go back to [3]. For more information concerning (3.11) see estimate (4.11) of [13]. Results of the type (3.12), (3.13) (with less precise right-hand side in (3.13)) go back to [11]. Estimate (3.13) follows, for example, from formulas (3.2), (3.3) and the estimate

$$
\begin{array}{r}
\left\|\Lambda^{-s} g(k) \Lambda^{-s}\right\|_{\mathbb{L}^{2}\left(\mathbb{R}^{d}\right) \rightarrow \mathbb{L}^{2}\left(\mathbb{R}^{d}\right)}=O\left(|k|^{-1}\right) \\
\text { as }|k| \rightarrow \infty, \quad k \in \mathbb{C}^{d} \backslash \mathbb{R}^{d},
\end{array}
$$

for $s>1 / 2$, where $g(k)$ denotes the integral operator with the Schwartz kernel $g(x-y, k)$ and $\Lambda$ denotes the multiplication operator by the function $\left(1+|x|^{2}\right)^{1 / 2}$. 
Estimate (3.15) was formulated, first, in [17] for $d \geq 3$. Concerning proof of (3.15), see [37].

In addition, we have that:

$$
\begin{aligned}
& h_{2}(k, l)-h_{1}(k, l)=(2 \pi)^{-d} \int_{\mathbb{R}^{d}} \psi_{1}(x,-l)\left(v_{2}(x)-v_{1}(x)\right) \psi_{2}(x, k) d x \\
& \text { for }(k, l) \in \Theta_{E},|\operatorname{Im} k|=|\operatorname{Im} l| \neq 0, \\
& \text { and } v_{1}, v_{2} \text { satisfying (3.5), } \\
& h_{2}(k, l)-h_{1}(k, l)=(2 \pi)^{-d} \int_{\partial D} \psi_{1}(x,-l)\left[\left(\hat{\Phi}_{2}-\hat{\Phi}_{1}\right) \psi_{2}(\cdot, k)\right](x) d x \\
& \text { for }(k, l) \in \Theta_{E},|\operatorname{Im} k|=|\operatorname{Im} l| \neq 0, \\
& \text { and } v_{1}, v_{2} \text { satisfying (1.5), (3.6), }
\end{aligned}
$$

and, under assumtions of Theorem 2.1,

$$
\begin{array}{r}
\left|\hat{v}_{1}(p)-\hat{v}_{2}(p)-h_{1}(k, l)+h_{2}(k, l)\right| \leq \frac{c_{2}(D, m) N\left\|v_{1}-v_{2}\right\|_{\mathbb{L}^{\infty}(D)}}{\left(E+\rho^{2}\right)^{1 / 2}} \\
\text { for }(k, l) \in \Theta_{E}, \quad p=k-l, \quad|\operatorname{Im} k|=|\operatorname{Im} l|=\rho, \\
E+\rho^{2} \geq r_{3}(N, D, m), \quad p^{2} \leq 4\left(E+\rho^{2}\right),
\end{array}
$$

where $h_{j}, \psi_{j}$ denote $h$ and $\psi$ of (3.3) and (3.2) for $v=v_{j}$, and $\hat{\Phi}_{j}$ denotes the Dirichlet-to-Neumann map for $v=v_{j}$, where $j=1,2$.

Formulas (3.16), (3.17) were given in [24], [27]. Estimate (3.18) follows from (3.2), (3.15), (3.16) in a similar way as estimate (3.13) follows from (3.2), (3.3), (3.15).

\section{Proof of Theorem 2.1}

Let

$$
\begin{aligned}
& \mathbb{L}_{\mu}^{\infty}\left(\mathbb{R}^{d}\right)=\left\{u \in \mathbb{L}^{\infty}\left(\mathbb{R}^{d}\right):\|u\|_{\mu}<+\infty\right\} \\
& \|u\|_{\mu}=\operatorname{ess} \sup _{p \in \mathbb{R}^{d}}(1+|p|)^{\mu}|u(p)|, \quad \mu>0 .
\end{aligned}
$$

Note that

$$
\begin{array}{r}
w \in \mathbb{W}^{m, 1}\left(\mathbb{R}^{d}\right) \Longrightarrow \hat{w} \in \mathbb{L}_{\mu}^{\infty}\left(\mathbb{R}^{d}\right) \cap \mathcal{C}\left(\mathbb{R}^{d}\right), \\
\|\hat{w}\|_{\mu} \leq c_{3}(m, d)\|w\|_{m, 1} \quad \text { for } \quad \mu=m,
\end{array}
$$

where $\mathbb{W}^{m, 1}, \mathbb{L}_{\mu}^{\infty}$ are the spaces of (2.2), (4.1),

$$
\hat{w}(p)=(2 \pi)^{-d} \int_{\mathbb{R}^{d}} e^{i p x} w(x) d x, \quad p \in \mathbb{R}^{d} .
$$

Using the inverse Fourier transform formula

$$
w(x)=\int_{\mathbb{R}^{d}} e^{-i p x} \hat{w}(p) d p, \quad x \in \mathbb{R}^{d},
$$


we have that

$$
\begin{array}{r}
\left\|v_{1}-v_{2}\right\|_{\mathbb{L}^{\infty}(D)} \leq \sup _{x \in \bar{D}}\left|\int_{\mathbb{R}^{d}} e^{-i p x}\left(\hat{v}_{2}(p)-\hat{v}_{1}(p)\right) d p\right| \leq \\
\leq I_{1}(r)+I_{2}(r) \quad \text { for any } r>0
\end{array}
$$

where

$$
\begin{aligned}
& I_{1}(r)=\int_{|p| \leq r}\left|\hat{v}_{2}(p)-\hat{v}_{1}(p)\right| d p, \\
& I_{2}(r)=\int_{|p| \geq r}\left|\hat{v}_{2}(p)-\hat{v}_{1}(p)\right| d p .
\end{aligned}
$$

Using (4.2), we obtain that

$$
\left|\hat{v}_{2}(p)-\hat{v}_{1}(p)\right| \leq 2 c_{3}(m, d) N(1+|p|)^{-m}, \quad p \in \mathbb{R}^{d} .
$$

Due to (3.18), we have that

$$
\begin{array}{r}
\left|\hat{v}_{2}(p)-\hat{v}_{1}(p)\right| \leq\left|h_{2}(k, l)-h_{1}(k, l)\right|+\frac{c_{2}(D, m) N\left\|v_{1}-v_{2}\right\|_{L^{\infty}(D)}}{\left(E+\rho^{2}\right)^{1 / 2}}, \\
\text { for }(k, l) \in \Theta_{E}, \quad p=k-l, \quad|\operatorname{Im} k|=|\operatorname{Im} l|=\rho, \\
E+\rho^{2} \geq r_{3}(N, D, m), \quad p^{2} \leq 4\left(E+\rho^{2}\right) .
\end{array}
$$

Let

$$
\begin{array}{r}
c_{4}=(2 \pi)^{-d} \int_{\partial D} d x, \quad L=\max _{x \in \partial D}|x|, \\
\delta=\left\|\hat{\Phi}_{2}(E)-\hat{\Phi}_{1}(E)\right\|,
\end{array}
$$

where $\left\|\hat{\Phi}_{2}(E)-\hat{\Phi}_{1}(E)\right\|$ is defined according to $(2.5)$.

Due to (3.17), we have that

$$
\begin{array}{r}
\left|h_{2}(k, l)-h_{1}(k, l)\right| \leq c_{4}\left\|\psi_{1}(\cdot,-l)\right\|_{\mathbb{L}^{\infty}(\partial D)} \delta\left\|\psi_{2}(\cdot, k)\right\|_{\mathbb{L}^{\infty}(\partial D)}, \\
(k, l) \in \Theta_{E},|\operatorname{Im} k|=|\operatorname{Im} l| \neq 0 .
\end{array}
$$

Using (3.11), we find that

$$
\begin{array}{r}
\|\psi(\cdot, k)\|_{\mathbb{L}^{\infty}(\partial D)} \leq \sigma \exp (|\operatorname{Im} k| L), \\
k \in \Sigma_{E},|k| \geq r_{1}(N, D, m, \sigma) .
\end{array}
$$

Here and bellow in this section the constant $\sigma$ is the same that in (3.11).

Combining (4.10) and (4.11), we obtain that

$$
\begin{array}{r}
\left|h_{2}(k, l)-h_{1}(k, l)\right| \leq c_{4} \sigma^{2} e^{2 \rho L} \delta, \quad \text { for }(k, l) \in \Theta_{E}, \\
\rho=|\operatorname{Im} k|=|\operatorname{Im} l|, \\
E+\rho^{2} \geq r_{1}^{2}(N, D, m, \sigma) .
\end{array}
$$

Using (4.8), (4.12), we get that

$$
\begin{array}{r}
\left|\hat{v}_{2}(p)-\hat{v}_{1}(p)\right| \leq c_{4} \sigma^{2} e^{2 \rho L} \delta+\frac{c_{2}(D, m) N\left\|v_{1}-v_{2}\right\|_{\mathbb{L}^{\infty}(D)}}{\left(E+\rho^{2}\right)^{1 / 2}}, \\
p \in \mathbb{R}^{d}, p^{2} \leq 4\left(E+\rho^{2}\right), E+\rho^{2} \geq \max \left\{r_{1}^{2}, r_{3}\right\} .
\end{array}
$$


Let

$$
\varepsilon=\left(\frac{1}{2 c_{2}(D, m) N c_{5}}\right)^{1 / d}, \quad c_{5}=\int_{p \in \mathbb{R}^{d},|p| \leq 1} d p,
$$

and $r_{4}(N, D, m, \sigma)>0$ be such that

$$
E+\rho^{2} \geq r_{4}(N, D, m, \sigma) \Longrightarrow\left\{\begin{array}{l}
E+\rho^{2} \geq r_{1}^{2}(N, D, m, \sigma), \\
E+\rho^{2} \geq r_{3}(N, D, m), \\
\left(\varepsilon\left(E+\rho^{2}\right)^{\frac{1}{2 d}}\right)^{2} \leq 4\left(E+\rho^{2}\right) .
\end{array}\right.
$$

Let

$$
c_{6}=\int_{p \in \mathbb{R}^{d},|p|=1} d p .
$$

Using (4.6), (4.13), we get that

$$
\begin{array}{r}
I_{1}(r) \leq c_{5} r^{d}\left(c_{4} \sigma^{2} e^{2 \rho L} \delta+\frac{c_{2}(D, m) N\left\|v_{1}-v_{2}\right\|_{\mathbb{L}^{\infty}(D)}}{\left(E+\rho^{2}\right)^{1 / 2}}\right), \\
r>0, r^{2} \leq 4\left(E+\rho^{2}\right), \\
E+\rho^{2} \geq r_{4}(N, D, m, \sigma) .
\end{array}
$$

Using (4.6), (4.7), we find that, for any $r>0$,

$$
I_{2}(r) \leq 2 c_{3}(m, d) N c_{6} \int_{r}^{+\infty} \frac{d t}{t^{m-d+1}} \leq \frac{2 c_{3}(m, D) N c_{6}}{m-d} \frac{1}{r^{m-d}} .
$$

Combining (4.5), (4.17), (4.18) for $r=\varepsilon\left(E+\rho^{2}\right)^{\frac{1}{2 d}}$ and (4.15), we get that

$$
\begin{array}{r}
\left\|v_{1}-v_{2}\right\|_{\mathbb{L}^{\infty}(D)} \leq c_{7}(N, D, m, \sigma) \sqrt{E+\rho^{2}} e^{2 \rho L} \delta+ \\
+c_{8}(N, D, m)\left(E+\rho^{2}\right)^{-\frac{m-d}{2 d}}+\frac{1}{2}\left\|v_{1}-v_{2}\right\|_{\mathbb{L}^{\infty}(D)}, \\
E+\rho^{2} \geq r_{4}(N, D, m, \sigma) .
\end{array}
$$

Let $\tau^{\prime} \in(0,1)$ and

$$
\beta=\frac{1-\tau^{\prime}}{2 L}, \quad \rho=\beta \ln \left(3+\delta^{-1}\right),
$$

where $\delta$ is so small that $E+\rho^{2} \geq r_{4}(N, D, m, \sigma)$. Then due to (4.19), we have that

$$
\begin{aligned}
& \frac{1}{2}\left\|v_{1}-v_{2}\right\|_{\mathbb{L}^{\infty}(D)} \leq \\
& \leq c_{7}(N, D, m, \sigma)\left(E+\left(\beta \ln \left(3+\delta^{-1}\right)\right)^{2}\right)^{1 / 2}\left(3+\delta^{-1}\right)^{2 \beta L} \delta+ \\
& \quad+c_{8}(N, D, m)\left(E+\left(\beta \ln \left(3+\delta^{-1}\right)\right)^{2}\right)^{-\frac{m-d}{2 d}}= \\
& =c_{7}(N, D, m, \sigma)\left(E+\left(\beta \ln \left(3+\delta^{-1}\right)\right)^{2}\right)^{1 / 2}(1+3 \delta)^{1-\tau^{\prime}} \delta^{\tau^{\prime}}+ \\
& +c_{8}(N, D, m)\left(E+\left(\beta \ln \left(3+\delta^{-1}\right)\right)^{2}\right)^{-\frac{m-d}{2 d}}
\end{aligned}
$$


where $\tau^{\prime}, \beta$ and $\delta$ are the same as in (4.20).

Using (4.21), we obtain that

$$
\left\|v_{1}-v_{2}\right\|_{\mathbb{L}^{\infty}(D)} \leq c_{9}\left(N, D, E, m, \sigma, \tau^{\prime}\right)\left(\ln \left(3+\delta^{-1}\right)\right)^{-\frac{m-d}{d}}
$$

for $\delta=\left\|\hat{\Phi}_{2}-\hat{\Phi}_{1}\right\| \leq \delta_{1}\left(N, D, E, m, \sigma, \tau^{\prime}\right)$, where $\delta_{1}$ is a sufficiently small positive constant. Estimate (4.22) in the general case (with modified $c_{9}$ ) follows from (4.22) for $\delta \leq \delta_{1}\left(N, D, E, m, \sigma, \tau^{\prime}\right)$ and the property that

$$
\left\|v_{j}\right\|_{\mathbb{L}^{\infty}(D)} \leq c_{10}(D, m) N .
$$

This completes the proof of $(2.8)$.

If $E \geq 0$ then there is a constant $\delta_{2}=\delta_{2}\left(N, D, m, \sigma, \tau^{\prime}\right)>0$ such that

$$
\delta \in\left(0, \delta_{2}\right) \Longrightarrow\left\{\begin{array}{l}
E+\left(\beta \ln \left(3+\delta^{-1}\right)\right)^{2} \geq r_{4}(N, D, m, \sigma), \\
E+\left(\beta \ln \left(3+\delta^{-1}\right)\right)^{2} \leq\left((1+\sqrt{E}) \beta \ln \left(3+\delta^{-1}\right)\right)^{2}, \\
\beta \ln \left(3+\delta^{-1}\right) \geq 1,
\end{array}\right.
$$

where $\beta$ is the same as in (4.20). Combining (4.21), (4.24), we obtain that for $s \in[0,(m-d) / d], \tau \in\left(0, \tau^{\prime}\right)$ and $\delta \in\left(0, \delta_{2}\right)$ the following estimate holds:

$$
\left\|v_{2}-v_{1}\right\|_{L^{\infty}(D)} \leq c_{11}(1+\sqrt{E}) \delta^{\tau}+c_{12}(1+\sqrt{E})^{s-\frac{m-d}{d}}\left(\ln \left(3+\delta^{-1}\right)\right)^{-s},
$$

where constants $c_{11}, c_{12}>0$ depend only on $N, D, m, \sigma, \tau^{\prime}$ and $\tau$.

Estimate (4.25) in the general case (with modified $c_{11}$ and $c_{12}$ ) follows from (4.25) for $\delta \leq \delta_{2}\left(N, D, m, \sigma, \tau^{\prime}\right)$ and (4.23).

This completes the proof of $(2.9)$

\section{Acknowledgements}

The second author was partially supported by the Russian Federation Goverment grant No. 2010-220-01-077.

\section{References}

[1] G. Alessandrini, Stable determination of conductivity by boundary measurements, Appl.Anal. 27, 1988, 153-172.

[2] G. Alessandrini, S. Vassella, Lipschitz stability for the inverse conductivity problem, Adv. in Appl. Math. 35, 2005, no.2, 207-241.

[3] R. Beals and R. Coifman, Multidimensional inverse scattering and nonlinear partial differential equations, Proc. Symp. Pure Math., 43, 1985, 45-70.

[4] L. Beilina, M.V. Klibanov, Approximate global convergence and adaptivity for coefficient inverse problems, Springer (New York), 2012. 407 pp.

[5] A. L. Buckhgeim, Recovering a potential from Cauchy data in the twodimensional case, J. Inverse Ill-Posed Probl. 16, 2008, no. 1, 19-33. 
[6] Calderón, A.P., On an inverse boundary problem, Seminar on Numerical Analysis and its Applications to Continuum Physics, Soc. Brasiliera de Matematica, Rio de Janeiro, 1980, 61-73.

[7] V. Druskin, The unique solution of the inverse problem in electrical surveying and electrical well logging for piecewise-constant conductivity, Physics of the Solid Earth 18(1), 1982, 51-53.

[8] L.D. Faddeev, Growing solutions of the Schrödinger equation, Dokl. Akad. Nauk SSSR, 165, N.3, 1965, 514-517 (in Russian); English Transl.: Sov. Phys. Dokl. 10, 1966, 1033-1035.

[9] L.D. Faddeev, The inverse problem in the quantum theory of scattering. II, Current problems in mathematics, Vol. 3, 1974, pp. 93180, 259. Akad. Nauk SSSR Vsesojuz. Inst. Naucn. i Tehn. Informacii, Moscow(in Russian); English Transl.: J.Sov. Math. 5, 1976, 334-396.

[10] I.M. Gelfand, Some problems of functional analysis and algebra, Proceedings of the International Congress of Mathematicians, Amsterdam, 1954, pp.253-276.

[11] G.M. Henkin and R.G. Novikov, The $\bar{\partial}$-equation in the multidimensional inverse scattering problem, Uspekhi Mat. Nauk 42(3), 1987, 93-152 (in Russian); English Transl.: Russ. Math. Surv. 42(3), 1987, 109-180.

[12] M.I. Isaev, Exponential instability in the Gelfand inverse problem on the energy intervals, J. Inverse Ill-Posed Probl., Vol. 19(3), 2011, 453-473; eprint arXiv: 1012.2193.

[13] M.I. Isaev, R.G. Novikov Stability estimates for determination of potential from the impedance boundary map, e-print arXiv:1112.3728.

[14] M.I. Isaev, R.G. Novikov Reconstruction of a potential from the impedance boundary map, e-print arXiv:1204.0076.

[15] V. Isakov, Increasing stability for the Schrödinger potential from the Dirichlet-to-Neumann map, Discrete Contin. Dyn. Syst. Ser. S 4, 2011, no. 3, 631-640.

[16] R. Kohn, M. Vogelius, Determining conductivity by boundary measurements II, Interior results, Comm. Pure Appl. Math. 38, 1985, 643-667.

[17] R.B. Lavine and A.I. Nachman, On the inverse scattering transform of the $n$-dimensional Schrödinger operator Topics in Soliton Theory and Exactly Solvable Nonlinear Equations ed M Ablovitz, B Fuchssteiner and M Kruskal (Singapore: World Scientific), 1987, pp 33-44.

[18] M.M. Lavrentev, V.G. Romanov, S.P. Shishatskii, Ill-posed problems of mathematical physics and analysis, Translated from the Russian by J. R. Schulenberger. Translation edited by Lev J. Leifman. Translations of Mathematical Monographs, 64. American Mathematical Society, Providence, RI, 1986. vi+290 pp. 
[19] L. Liu, Stability Estimates for the Two-Dimensional Inverse Conductivity Problem, Ph.D. thesis, Department of Mathematics, University of Rochester, New York, 1997.

[20] N. Mandache, Exponential instability in an inverse problem for the Schrödinger equation, Inverse Problems. 17, 2001, 1435-1444.

[21] A. Nachman, Global uniqueness for a two-dimensional inverse boundary value problem, Ann. Math. 143, 1996, 71-96.

[22] S. Nagayasu, G. Uhlmann, J.-N. Wang, Increasing stability in an inverse problem for the acoustic equation, e-print arXiv:1110.5145

[23] R.G. Novikov, Multidimensional inverse spectral problem for the equation $-\Delta \psi+(v(x)-E u(x)) \psi=0$ Funkt. Anal. Prilozhen. 22(4), 1988, 11-22 (in Russian); Engl. Transl. Funct. Anal. Appl. 22, 1988, 263-272.

[24] R.G. Novikov, $\bar{\partial}$-method with nonzero background potential. Application to inverse scattering for the two-dimensional acoustic equation, Comm. Partial Differential Equations 21, 1996, no. 3-4, 597-618.

[25] R.G. Novikov, Rapidly converging approximation in inverse quantum scattering in dimension 2, Physics Letters A 238, 1998, 73-78.

[26] R.G. Novikov, The $\bar{\partial}$-approach to approximate inverse scattering at fixed energy in three dimensions. IMRP Int. Math. Res. Pap. 2005, no. 6, 287349 .

[27] R.G. Novikov, Formulae and equations for finding scattering data from the Dirichlet-to-Neumann map with nonzero background potential, Inverse Problems 21, 2005, 257-270.

[28] R.G. Novikov, The $\bar{\partial}$-approach to monochromatic inverse scattering in three dimensions, J. Geom. Anal 18, 2008, 612-631.

[29] R.G. Novikov, New global stability estimates for the Gel'fand-Calderon inverse problem, Inverse Problems 27, 2011, 015001(21pp); e-print arXiv:1002.0153.

[30] R. Novikov and M. Santacesaria, A global stability estimate for the GelfandCalderon inverse problem in two dimensions, J.Inverse Ill-Posed Probl., Volume 18, Issue 7, 2010, Pages 765-785; e-print arXiv: 1008.4888.

[31] R. Novikov and M. Santacesaria, Monochromatic Reconstruction Algorithms for Two-dimensional Multi-channel Inverse Problems, International Mathematics Research Notes, 2012, doi: 10.1093/imrn/rns025.

[32] V.P. Palamodov, private communication of February 2011.

[33] L. Rondi, A remark on a paper by Alessandrini and Vessella, Adv. in Appl. Math. 36 (1), 2006, 67-69.

[34] M. Santacesaria, New global stability estimates for the Calderon inverse problem in two dimensions, e-print: hal-00628403. 
[35] M. Santacesaria, Stability estimates for an inverse problem for the Schrödinger equation at negative energy in two dimensions, e-print: hal00688457.

[36] J. Sylvester and G. Uhlmann, A global uniqueness theorem for an inverse boundary value problem, Ann. of Math. 125, 1987, 153-169.

[37] R. Weder, Generalized limiting absorption method and multidimensional inverse scattering theory, Mathematical Methods in the Applied Sciences, 14, 1991, 509-524.

\section{M.I. Isaev}

Centre de Mathématiques Appliquées, Ecole Polytechnique, 91128 Palaiseau, France

Moscow Institute of Physics and Technology,

141700 Dolgoprudny, Russia

e-mail: isaev.m.i@gmail.com

\section{R.G. Novikov}

Centre de Mathématiques Appliquées, Ecole Polytechnique,

91128 Palaiseau, France

Institute of Earthquake Prediction Theory and Math. Geophysics RAS,

117997 Moscow, Russia

e-mail: novikov@cmap.polytechnique.fr 